\title{
Words and pronunciation as coding aids ${ }^{1,2}$
}

RICHARD H. LINDLEY

In Experiment I after an initial presentation of 1 or 2 to-berecalled trigrams, E either spelled the items, pronounced them, or said related words. Pronounciation did not facilitate recall, whereas the related words did. Experiment II showed that the failure to find facilitatory effects of pronounciation in Experiment I was due to whether $E$ or $S$ did the spelling and pronouncing.

Mechanic \& D'Andrea (1966, p. 343) assert that "overt or covert pronouncing responses to verbal items can greatly facilitate response learning." Schaub \& Lindley (1964) found that pairing related words with trigrams (e.g., HONEY-HON) facilitated short-term memory (STM) for the trigrams. Perhaps the words make the trigrams easier to pronounce; Experiment I compared words and pronouncing as memory aids.

\section{EXPERIMENT I}

\section{Method}

The between-Ss factor of the 3 by 3 factorial design was 3 types of items (1 trigram presented twice, e.g., CAG CAG; 2 trigrams with immediate repetition, e.g., CAG CAG ZER ZER; 2 trigrams with delayed repetition, e.g., CAG ZER CAG ZER). The within-Ss factor was 3 different activities performed by $E$ after the 1st spelling of the item (spell items again, pronounce items, or say 1 or 2 words. The 1st 3 letters of the word always formed the trigram, e.g., CAG Cage ZER Zero; this was explained to S).

The items were presented in 3 blocks of 6 items with each block corresponding to the spell, pronounce, or word activities of $E$. The order of items within a block was constant; over Ss each block appeared equally often as the 1st, 2nd, 3rd set of items.

E presented the items aurally to 63 randomly assigned college student Ss in time to a 96 beats $/ \mathrm{min}$. metronome: E said "Ready"'; paused 3 beats; spelled the item; then (depending on the condition), either spelled the item again, pronounced it, or said the word; and then said a 3-digit number. $S$ repeated the number and subtracted backwards by $3 \mathrm{~s}$ for $26 \mathrm{sec}$. $S$ had $20 \mathrm{sec}$. to recall the items and then had a $5 \mathrm{sec}$. rest. $\mathrm{E}$ paused for 1 beat between each set of 3 letters and between each pronunciation or word indicating that each trigram was a unit.

\section{Results and Discussion}

In scoring correct recalls credit was given for each letter correctly recalled at the proper serial position; order of trigram recall was ignored (e.g., for CAG ZER, ZEB RAG or RAG ZEB would be scored as 4 letters correct). The left-hand portion of Fig. 1 presents the data.
Not surprisingly, more letters were recalled correctly with the six-letter than with the three-letter items $(F=61.07, d f=1 / 60, p<.01)$; however, there was no difference between immediate and delayed trigram repetition ( $F=0, d f=1 / 60$ ). There was also no difference between $E$ spelling or pronouncing the items $(F=0$, df $=1 / 120$ ) while the item-word condition was clearly superior to the item-spell and item-pronounce conditions $(F=115.01, d f=1 / 120, p<.01)$. Only one of the main effect interactions had an F>1; it was three- vs. six-letter items by words vs. spell-pronounce $(F=29.70$, $\mathrm{df}=1 / 120, p<.01$ ). This interaction was significant because words improved recall more for six-letter than for three-letter items; this was due to a "ceiling" effect since recall of the three-letter items when spelled was so high that there was little room for improvement when words were given.

In the present study word coding aids but not pronunciation responses aided STM. Thus the facilitatory effects of word coding aids on STM are not reducible to increases in pronunciability brought about by the words. Mechanic \& D'Andrea (1966) found that pronunciation aids paired-associate learning; however, the positive result was obtained when $S$ did the pronouncing while the negative result of the present study was obtained when $\mathrm{E}$ did the pronouncing; Experiment II compared the effects of $E$ and $S$ pronunciation on STM.

\section{EXPERIMENT II}

Underwood \& Schulz (1960) presented evidence that ease of learning is a function of trigram pronunciability. Pronunciation could aid learning by suggesting word

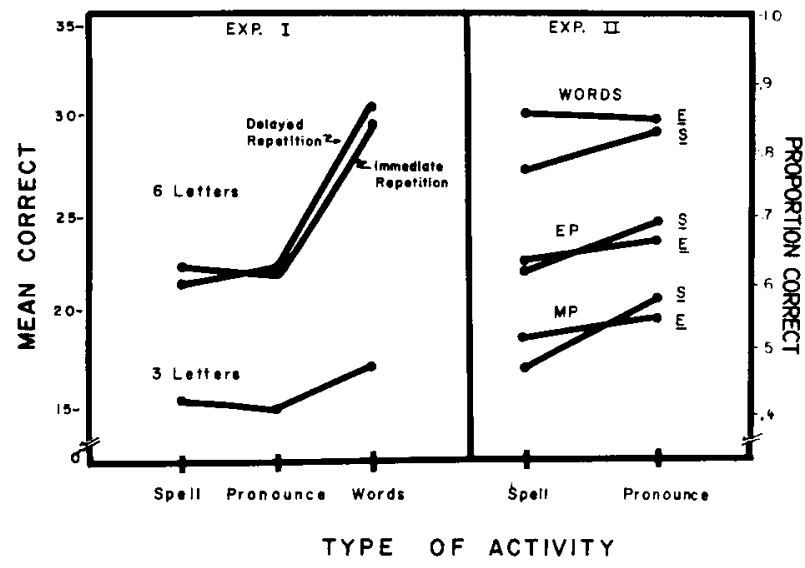

Fig. 1. Recall as a function of type of activity. (Proportions correctly recalled should be doubled for 3-letter items.) 
associations for the trigrams which enable $S$ to reduce the number of chunks of information in the message; e.g., pronouncing the three letters A-B-L may suggest the single chunk ABLE. This explanation implies that chunking through pronunciation should be easier to accomplish with high meaningfulness (M) than with low $M$ trigrams since pronunciation should suggest more words for the former items than for the latter. Method

The between-Ss factor of the 3 by 2 by 2 factorial design was level of $M$ (words, easy-pronounce (EP) trigrams, medium pronunciability (MP) trigrams). The within-Ss factors were spelling or pronouncing the to-be-recalled item aloud after the initial aural presentation and who performed this activity ( $E$ or $S$ ); $S$ was given sufficient time to complete his spelling or pronunciation before the 3-digit number was presented. The delayed repetition STM procedure described in Experiment I was followed except that $10 \mathrm{sec}$. was allowed for recall.

All items were six letters long; the words were composed of two three-letter words (e.g., CAR, HOW). The EP and MP items were composed of two EP or MP trigrams (e.g., MUL FON, ABL VUF) from Appendix E of Underwood \& Schulz (1960); the mean MP PR was 4.9, EP was 2.9. The 108 college student Ss were assigned randomly (with correction for equal Ns) to one of the three levels of $M$.

All Ss recalled 24 items at one level of $M$. There were 4 blocks of 6 items each; the order of presentation of the items within a block and the order of presentation of blocks were counterbalanced over Ss. Results and Discussion

The right-hand portion of Fig. 1 shows the correct recall data. Analysis of variance showed that $M$ was a significant source of variance $(F=34.95, \mathrm{df}=2 / 105$, $\mathrm{p}<.01)$. Pronouncing the items after presentation proved superior to spelling $(\mathrm{F}=15.03, \mathrm{df}=1 / 315, \mathrm{p}<.01)$. Who performed the spelling or pronouncing ( $E$ or $S$ ) approached statistical significance $(F=3.17, d f=1 / 315$, $\mathrm{p}<.10>.05$ ) although, as inspection of Fig. 1 shows, the relationship is complex. The pronounce-spell by $E-S$ interaction was also significant $(F=6.14, d f=1 / 315$, $\mathrm{p}<.02)$.

None of the interactions of the main effects with $M$ was statistically significant; therefore, the following conclusions are based on data combined for the three levels of $M$. The significant effects of pronunciation and of the spell-pronounce by $E-S$ interaction derive from the poor performance for $S$ spelling. (The mean number correctly recalled for the three levels of $M$ combined for $\mathrm{E}$ pronounce, $\mathrm{E}$ spell, S pronounce, and $\mathrm{S}$ spell was $24.5,24.1,25.0,22.2$, respectively.) An obvious explanation of this finding is that $\mathrm{S}$ spelling prevents $S$ from pronouncing the to-be-recalled item; when $E$ spells the item twice, there is time available for $S$ to pronounce the item silently during the second spelling. This finding supports the hypothesis that the different findings of Mechanic and Lindley are due to whether $\mathrm{E}$ or $\mathrm{S}$ pronounces the item.

As pointed out in the introduction, reducing the number of chunks by coding with word associations should be more effective with EP than with MP items since more words can be given to EP than to MP items. The interactions of $\mathrm{E}-\mathrm{S}$ by $\mathrm{M}$, spell-pronounce by $\mathrm{M}$, and $E-S$ by spell-pronounce by $M$ were not significant $(F=1.38,<1,<1$, respectively, df $=2 / 315$ ); thus, pronunciation was not more effective than spelling at the higher levels of $M$. These results suggest that the facilitation of STM by S pronunciation as compared to $S$ spelling is not due to the word association coding mechanism described here.

Comparing the results of Experiments I and II (see Fig. 1) shows that the 6-letter items of Experiment I were (in terms of their recallability) approximately at the EP level of meaningfulness of the Experiment II items. Pronunciation by $S$ in Experiment II had only a small facilitative effect on STM whereas pairing related words with trigrams in Experiment I had a very large facilitative effect on STM. Thus coding with words was much more effective as a memory aid than was coding by pronunciation.

\section{References}

Mechanic, A., \& D'Andrea, J. Visual and pronouncing responses, and the relation between orienting task and presentations in incidental learning. J. exp. Psychol., 1966, 71, 343-349.

Schaub, G. R., \& Lindley, R. H. Effects of subject-generated recoding cues on short-term memory. J. exp. Psychol., 1964, $68,171-175$.

Underwood, B. J., \& Schulz, R. W. Meaningfulness and verbal learning. Philadelphia: Lippincott, 1960.

\section{Notes}

1. Supported by a National Science Foundation Research Grant. 2. Daniel Edwards, Ruth Boyatt, Bernard Ostrowski, David Brooks, and William John provided very capable assistance in carrying out these studies. 